\title{
Article
}

\section{Percutaneous Mitral Valve Repair in Patients with Severe Mitral Regurgitation and Acute Decompensated Heart Failure}

\author{
Anna Turyan Medvedovsky ${ }^{1,+}$, Dan Haberman ${ }^{2,+}{ }^{\oplus}$, Mahsati Ibrahimli ${ }^{3}$, Ivaylo Tonchev ${ }^{3} \mathbb{D}$, Yonatan Rashi ${ }^{1}$, \\ Alona Peretz ${ }^{3}$, Sara Shimoni ${ }^{2}$, Ortal Tuvali ${ }^{2}$, Haim Danenberg ${ }^{4}$, Ronen Beeri ${ }^{3}$ and Mony Shuvy ${ }^{1, *}$ \\ 1 The Jesselson Integrated Heart Center, Shaare Zedek Medical Center, Shmu'el Bait St. 12, Jerusalem 9103102 , \\ Israel; drannia2004@gmail.com (A.T.M.); yrashi1@gmail.com (Y.R.) \\ 2 Kaplan Heart Center, Kaplan Medical Center, Faculty of Medicine, Hebrew University of Jerusalem, \\ Pasternak St. P.O. Box 1, Rehovot 76100, Israel; haberdan@gmail.com (D.H.); sarah_s2@clalit.org.il (S.S.); \\ ortalhagege@gmail.com (O.T.) \\ 3 Heart Institute, Hadassah-Hebrew University Medical Center, Jerusalem 90000, Israel; \\ mehseti.ibrahimli@gmail.com (M.I.); ivailortonchev@gmail.com (I.T.); alonaperetz@gmail.com (A.P.); \\ ronenbe@ekmd.huji.ac.il (R.B.) \\ 4 Heart Institute, Wolfson Medical Center, Ha-Lokhamim St. 62, Holon 5822012, Israel; danen040@gmail.com \\ * Correspondence: monysh@gmail.com; Tel./Fax: +972-2655-5975 \\ + Equal contribution.
}

\section{check for} updates

Citation: Turyan Medvedovsky, A.; Haberman, D.; Ibrahimli, M.; Tonchev, I.; Rashi, Y.; Peretz, A.; Shimoni, S.; Tuvali, O.; Danenberg, H.; Beeri,

R.; et al. Percutaneous Mitral Valve Repair in Patients with Severe Mitral Regurgitation and Acute Decompensated Heart Failure. J. Clin. Med. 2021, 10, 5849. https://doi.org/ $10.3390 /$ jcm10245849

Academic Editor: Patrick De Boever

Received: 16 September 2021

Accepted: 25 November 2021

Published: 13 December 2021

Publisher's Note: MDPI stays neutral with regard to jurisdictional claims in published maps and institutional affiliations.

Copyright: (c) 2021 by the authors. Licensee MDPI, Basel, Switzerland. This article is an open access article distributed under the terms and conditions of the Creative Commons Attribution (CC BY) license (https:/ / creativecommons.org/licenses/by/ $4.0 /)$.
Abstract: The role of percutaneous mitral valve repair (PMVr) in management of high-risk patients with severe mitral regurgitation (MR) and acute decompensated heart failure (ADHF) is undetermined. We screened all patients who underwent PMVr between October 2015 and March 2020. We evaluated immediate, 30-day, and 1-year outcomes in patients who underwent PMVr during hospitalization due to ADHF as compared to elective patients. From a cohort of 237 patients, we identified 46 patients (19.4\%) with severe MR of either functional or degenerative etiology who underwent PMVr during index hospitalization due to ADHF, including 17 (37\%) critically ill patients. Patients' mean age was $75.2 \pm 9.8$ years, $56 \%$ were males. There were no differences in background history between ADHF and elective patients. Patients with ADHF were at higher risk for surgery, reflected in higher mean EuroSCORE II, compared with elective patients. After PMVr, we observed higher 30-day mortality rate in ADHF patients as compared to the elective group (10.9\% vs. 3.1\%, respectively, $p=0.042)$. One-year mortality rate was similar between the groups $(21.7 \%$ vs. $17.9 \%$, $p=0.493)$. Clinical and echocardiographic follow-up showed improvement of NYHA functional class and sPAP reduction in both groups ( $(54 \pm 15 \mathrm{mmHg}$ to $50 \pm 15$ in the elective group $(p=0.02)$, $58 \pm 13 \mathrm{mmHg}$ to $52 \pm 12$ in the ADHF group $(p=0.02))$. PMVr could be an alternative option for treatment of patients with severe MR and ADHF.

Keywords: mitral regurgitation; acute decompensated heart failure; percutaneous mitral valve intervention

\section{Introduction}

Acute decompensated heart failure (ADHF) is associated with high rates of mortality and morbidity including frequent short-term unplanned hospitalizations [1,2]. The management of ADHF remains challenging. It is important to identify and treat specific precipitation factors that lead to ADHF. The most common precipitants are noncompliance with medications or dietary restrictions, uncontrolled hypertension, ischemia, arrhythmias, and exacerbation of chronic obstructive pulmonary disease [3].

Mitral regurgitation (MR) is the most common valvular heart disorder and a large cause of heart failure (HF) exacerbation [4,5]. In advanced HF, MR increases the burden of the failing ventricle and decreases effective stroke volume [6]. Both primary (degenerative) and secondary (functional) MR may lead to ADHF. The underling mechanism of degenerative MR in ADHF is disruption of any component of the apparatus or surrounding 
anatomy that can lead to MR (such as flail leaflet due to cord rupture) [4]. Functional MR is a ventricular disease that occurs despite a structurally normal mitral valve, which can be classified as having an ischemic, or non-ischemic etiology, the former being the most common and occurring after myocardial infarction (MI). The mechanism of acute ischemic MR complicating an acute MI includes the rupture of a papillary muscle or acute left ventricle (LV) remodeling, which leads to apical and posterior displacement of the papillary muscles, causing leaflet tethering and reduced closing forces $[7,8]$. The pre-dominant mechanism for non-ischemic functional MR is an increased effective regurgitant orifice from annular dilation and loss of annular contraction. It can be caused by idiopathic dilated cardiomyopathy (DCM), long-standing hypertension, and myocarditis [9]. Management of patients with ADHF and severe MR is often challenging.

The management of ADHF during index hospitalization is often different from that of chronic HF as in-patient treatment consists primarily of hemodynamic stabilization, symptom relief, and prevention of short-term morbidity and mortality [4]. The role of Percutaneous Mitral Valve repair (PMVr) is less known in the management of high-risk patients with ADHF and severe MR during index hospitalization. In our study, we evaluated the immediate, 30-day, and 1-year outcomes of PMVr in patients with ADHF and severe MR of both functional and degenerative etiology without adequate response to the optimal medical therapy.

\section{Materials and Methods}

\subsection{Study Population}

We screened all patients who underwent Transcatheter Edge-to-Edge Repair (TEER) with MitraClip ${ }^{\circledR}$ implantation at two medical centers (Hadassah Medical Center and Kaplan Medical Center) between October 2015 and March 2020. All patients received thorough verbal and written explanations about MitraClip implantation procedure and signed informed consents. The institutional committee for human studies of both centers approved the study protocol. We evaluated patients with severe MR who were hospitalized due to ADHF or electively, including a cohort of critically ill patients with refractory HF. Patients with ADHF presented with a rapid onset and worsening of symptoms of HF and New York Heart Association (NYHA) functional class above III, often in the context of pre-existing cardiomyopathy. The diagnosis of ADHF was based on clinical features that included pulmonary congestion and dependence on intravenous (IV) continuous infusions of diuretics (high dose of Furosemide). Critically ill patients with cardiogenic shock presented with low systolic blood pressure (below $90 \mathrm{mmHg}$ ), clinical signs of poor tissue perfusion (oligo/anuria, cyanosis, cool extremities, and altered mentation), and were dependent on inotropic/vasopressors drugs (Norepinephrine or Dopamine in inotropic dose) in order to increase blood pressure, and/or Intra-Aortic Balloon Pump (IABP) support. In our study, the IABP was placed only in post-MI patients with acute ischemic MR. Elective patients presented with stable HF symptoms (shortness of breath during regular or slight exertions) and scheduled for a planned procedure. The Heart Team that included HF cardiologist, Interventional cardiologist, Cardiac surgeon, and Interventional echocardiographer evaluated each case. TEER was performed in high-riskfor-surgery, symptomatic patients with moderate-to-severe or severe functional mitral regurgitation (MR) who remained symptomatic despite stable doses of maximally tolerated Guideline-Directed Medical Therapy (GDMT) plus cardiac resynchronization therapy, if appropriate, or for the treatment of significant symptomatic degenerative MR according to an FDA-approved indication.

\subsection{Echocardiographic Assessment}

Transthoracic (TTE) and transesophageal echocardiography (TEE) were performed to evaluate the severity of MR and to assess suitability for MitraClip ${ }^{\circledR}$ implantation. We used an available ultrasound diagnostic systems (Vivid 7 and Vivid E9, GE Medical Systems, Milwaukee, WI, USA and Philips IE 33, Royal Philips Electronics, Amsterdam, The 
Netherlands) with two-dimensional imaging and Doppler data including pulsed wave and continuous wave (CW) imaging. The severity of MR was initially assessed by integrated multiparametric visual evaluation tool in accordance with standard clinical practice (incorporating 2D, spectral and color Doppler images) using an ordinal scale (grading 0 -no MR, 1+ mild MR, 2+ moderate MR, 3+ moderate to severe MR, 4+ severe MR), and effective regurgitant orifice area (EROA) was calculated based on proximal isovelocity surface area (PISA) method with $40 \mathrm{~mm}^{2}$ as cutoff for severe MR [10]. The etiology of MR was evaluated according to the European Association of Echocardiography recommendations [11]. We evaluated patients with severe degenerative MR due to flail leaflet and ruptured cord as well as severe functional MR due to ischemic (after a recent MI) and non-ischemic etiology (secondary to DCM). Functional MR was defined in the case of abnormal function of normal leaflets in the context of impaired LV function, without echocardiographic signs of other intrinsic pathology of the leaflets, chordae, and papillary muscles [4]. Pulmonary artery systolic pressures (sPAP) were estimated by calculating maximal tricuspid pressure gradient, using CW Doppler, and the right atrial pressure based on inferior vena cava size and changes during inspiration. A sPAP $\leq 40 \mathrm{mmHg}$ was considered normal [11].

\subsection{Intervention}

Transcatheter edge-to-edge PMVr was performed by implantation of device "MitraClip ${ }^{\circledR}$ " (Abbott, Abbott Park, IL, USA) - a catheter-based system, which consists of a steerable 24 Fr guide catheter and a clip delivery system. The clip device system was delivered to the left atrium via a trans-septal puncture, then advanced into the LV and retracted grasping the mitral valve leaflets. The second or third clip device was placed at operator discretion to obtain additional MR reduction. All clips were implanted under general anesthesia and fluoroscopic and TEE guidance [12]. Procedural success was defined as MR reduction to grade $1+$ or $2+$ after implantation of at least one clip. All patients were transferred to our intensive care cardiac unit (ICCU) after the procedure for further observation.

\subsection{Outcomes}

We evaluated clinical outcomes including NYHA functional class, 30-day and 1-year mortality, and 1-year rehospitalization after PMVr in patients with severe MR admitted electively or due to ADHF. Additional outcomes included hemodynamic stabilization, echocardiographic assessment of MR, LV end-diastolic diameter (LVEDD), and sPAP after PMVr. For the follow-up assessment, all patients were scheduled for 30-day and 12-months as outpatients or telephone visits.

\subsection{Statistical Analysis}

Our analyses were performed using the entire cohort, comparing between patients presenting with ADHF or electively admitted. Patient characteristics are reported according to variable properties. Continuous variables were expressed as mean \pm standard deviation or median and interquartile range, where appropriate. Those with a normal distribution are reported as mean ( \pm standard deviation), and differences between subgroups were tested using the student's $t$-test. Those without a normal distribution are reported (interquartile range), and differences between subgroups were tested using the Mann-Whitney U. Categorical variables were presented as counts and percentages.

Follow-up time was calculated using Kaplan-Meier estimate of potential follow-up. Kaplan-Meier curves with the log-rank test were used to compare survival. All clinical events were analyzed by time to first event for Kaplan-Meier analysis. A two-tailed $p$-value $\leq 0.05$ is regarded as statistically significant. The IBM Statistical Package for the Social Sciences (SPSS) Statistics 26.0 (IBM Corp., Armonk, NY, USA) was utilized to perform the analyses. 


\section{Results}

From a cohort of 237 patients who underwent PMVr implantation in two centers, we identified 46 patients (19.4\%) with ADHF. The other 191 patients (81\%) were admitted electively. The mean patient age was $75 \pm 10$ year, $40 \%$ were females.

Baseline clinical characteristics of study population before PMVr are shown in Table 1.

Table 1. Baseline clinical characteristics of study population before PMVr.

\begin{tabular}{|c|c|c|c|c|}
\hline \multirow[b]{2}{*}{ Clinical Characteristics } & \multicolumn{3}{|c|}{ Patient Population } & \multirow[b]{2}{*}{$p$-Value } \\
\hline & $\begin{array}{l}\text { Total Population, } \\
\qquad n=237\end{array}$ & $\begin{array}{l}\text { Patients with ADHF, } \\
\qquad n=46\end{array}$ & $\begin{array}{c}\text { Elective Patients, } \\
\qquad n=191\end{array}$ & \\
\hline Age, years & $75.2 \pm 9.8$ & $73.0 \pm 9.4$ & $75.9 \pm 9.8$ & 0.08 \\
\hline Male, $n(\%)$ & $139(56)$ & $25(54)$ & $114(60)$ & 0.51 \\
\hline Known CAD, $n(\%)$ & $146(62)$ & $31(67)$ & $115(60)$ & 0.50 \\
\hline Post MI, $n(\%)$ & $90(38)$ & $21(46)$ & $69(36)$ & 0.24 \\
\hline Hypertension, $n(\%)$ & $204(86)$ & $40(86)$ & $164(87)$ & 1.00 \\
\hline Diabetes Mellitus, $n(\%)$ & $91(38)$ & $21(45)$ & $70(36)$ & 0.21 \\
\hline Hyperlipidemia, $n(\%)$ & $188(79)$ & $35(76)$ & $153(80)$ & 0.55 \\
\hline Smoker, $n(\%)$ & $88(37)$ & $18(39)$ & $70(37)$ & 0.41 \\
\hline $\mathrm{S} / \mathrm{P}$ CVA, $n(\%)$ & $44(19)$ & $8(17)$ & $36(19)$ & 1.00 \\
\hline $\mathrm{AFIB}, n(\%)$ & $113(48)$ & $23(51)$ & $90(47)$ & 0.34 \\
\hline Post-thoracotomy, $n(\%)$ & $65(27)$ & $6(13)$ & $59(31)$ & 0.03 \\
\hline $\begin{array}{l}\text { HF-related hospitalizations } \\
\text { last year, } \%\end{array}$ & $197(83)$ & $44(96)$ & $153(80)$ & $>0.01$ \\
\hline \multicolumn{5}{|l|}{ Cardiac-related drug therapy: } \\
\hline ACE-inhibitors or ARBs, $n(\%)$ & $169(71)$ & $32(69)$ & $137(72)$ & 0.17 \\
\hline Beta blockers, $n(\%)$ & $201(84)$ & $34(73)$ & $167(88)$ & 0.02 \\
\hline MRA, $n(\%)$ & $109(46)$ & $15(33)$ & $100(52)$ & 0.02 \\
\hline Furosemide, $n(\%)$ & $206(87)$ & $38(83)$ & $168(88)$ & 0.34 \\
\hline EuroSCORE II & $10.3 \pm 10.8$ & $15.9 \pm 13.1$ & $9.0 \pm 8.5$ & 0.01 \\
\hline $\mathrm{Hb}, \mathrm{g} / \mathrm{L}$ & $11.8 \pm 1.6$ & $12.0 \pm 1.7$ & $11.7 \pm 1.6$ & 0.46 \\
\hline $\mathrm{Cr}, \mathrm{mg} / \mathrm{dL}$ & $138 \pm 85$ & $111.8 \pm 41.8$ & $146.2 \pm 93.3$ & 0.1 \\
\hline $\mathrm{GFR}, \mathrm{mL} / \mathrm{min}$ & $53 \pm 24$ & $57.9 \pm 19.4$ & $51.2 \pm 25.4$ & 0.27 \\
\hline GFR $<30 \mathrm{~mL} / \mathrm{min}, n(\%)$ & $54(23)$ & $5(11)$ & $49(26)$ & 0.04 \\
\hline Mean NYHA functional class & $3.4 \pm 0.6$ & $3.7 \pm 0.8$ & $3.3 \pm 0.5$ & 0.01 \\
\hline NYHA functional class IV, $\%$ & $101(43)$ & $41(89)$ & $60(32)$ & 0.01 \\
\hline
\end{tabular}

$\mathrm{ADHF}$ = acute decompensated heart failure; $\mathrm{CAD}$ = coronary artery disease; $\mathrm{MI}$ = myocardial infarction; $\mathrm{CVA}$ = cerebrovascular accident; $\mathrm{AFIB}=$ atrial fibrillation; $\mathrm{HF}=$ heart failure; $\mathrm{ACE}=$ Angiotensin-converting enzyme; $\mathrm{ARB}=$ Angiotensin II receptor blockers; $\mathrm{MRA}=$ mineralocorticoid receptor antagonist; $\mathrm{Hb}=$ hemoglobin; $\mathrm{Cr}=$ creatinine; GFR = Glomerular filtration rate; NYHA = New York Heart Association

There were no differences in age and sex between ADHF and elective patients. There was no significant difference in background illnesses: Diabetes mellitus, Hypertension, Hyperlipidemia, history of smoking, and previous stroke between the two groups. There was a tendency toward higher prevalence of previous MI in the ADHF group compared with the elective group ( $45.7 \%$ vs. $36.1 \%, p=0.24)$. Patients presented with ADHF had significantly higher prevalence of HF-related hospitalizations during the last year as compared with elective patients $(95.7 \%$ vs. $80.0 \%, p=0.01)$. There were no significant differences in medical treatment between the two groups, except for lower use of beta-blockers and 
mineralocorticoid-receptor antagonists (MRA) in the ADHF group compared to the elective group [66.7 vs. $87 \%(p=0.02)$ and 33.3 vs. $52.3 \%(p=0.02)$, respectively]. Patients with ADHF were at higher risk for surgery reflected in higher mean EuroSCORE II, compared with elective patients $(15.9 \pm 13.1 \%$ vs. $9.0 \pm 8.5 \%$ respectively, $p=0.01)$. The prevalence of advanced renal failure with GFR $<30 \mathrm{~mL} / \mathrm{min}$ was lower in the ADHF group as compared with elective patients $(11 \%$ vs. $26 \%, p=0.04)$. ADHF patients were more likely to present with advanced HF and higher grade of NYHA functional class IV ( $84.4 \%$ vs. $31.2 \%, p=0.01)$ as compared to elective patients.

Out of 46 ADHF patients, 17 patients (37.0\%) were critically ill. All critically ill patients were dependent on IV infusions of inotropes/vasopressors prior to the PMVr in order to increase blood pressure despite MR exacerbation. Fifteen patients $(32.6 \%)$ with post-MI acute functional MR depended on IABP for mechanical support before the PMVr.

Baseline echocardiographic characteristic are shown in Table 2.

Table 2. Baseline echocardiographic characteristics of patient population before PMVr.

\begin{tabular}{|c|c|c|c|c|}
\hline \multirow{2}{*}{$\begin{array}{l}\text { Echocardiographic } \\
\text { Characteristics }\end{array}$} & \multicolumn{3}{|c|}{ Patient Population } & \multirow[b]{2}{*}{$p$-Value } \\
\hline & $\begin{array}{l}\text { Total Population, } \\
\qquad n=237\end{array}$ & $\begin{array}{c}\text { ADHF Patients, } \\
\qquad n=46\end{array}$ & $\begin{array}{l}\text { Elective Patients, } \\
\qquad n=191\end{array}$ & \\
\hline MR grade $3^{+}, n(\%)$ & $55(23)$ & $8(18)$ & $47(24)$ & \multirow{2}{*}{0.64} \\
\hline MR grade $4^{+}, n(\%)$ & $182(77)$ & $38(82)$ & $144(76)$ & \\
\hline EROA, $\mathrm{mm}^{2}(\mathrm{SD})$ & $41 \pm 11$ & $43 \pm 11$ & $41 \pm 10$ & 0.81 \\
\hline LVEF, \% (SD) & $35.5 \pm 13.4$ & $32.4 \pm 13.6$ & $36.2 \pm 13.3$ & 0.15 \\
\hline$<40 \%, n(\%)$ & $159(67)$ & $37(79)$ & $122(64)$ & \multirow{3}{*}{0.23} \\
\hline $40-50 \%, n(\%)$ & $34(14)$ & $4(9)$ & $30(16)$ & \\
\hline$>50 \%, n(\%)$ & $44(19)$ & $5(12)$ & $41(20)$ & \\
\hline LVEDD, mm (SD) & $57.4 \pm 10.2$ & $58.5 \pm 11.2$ & $57.2 \pm 9.9$ & 0.57 \\
\hline sPAP, mmHg (SD) & $54.5 \pm 15.0$ & $58.4 \pm 13.7$ & $53.5 \pm 15.2$ & 0.07 \\
\hline
\end{tabular}

$\mathrm{PMVr}=$ percutaneous mitral valve repair; $\mathrm{ADHF}=$ acute decompensated heart failure; $\mathrm{EROA}=$ effective regurgitant orifice area; $\mathrm{MR}=$ mitral regurgitation; $\mathrm{LVEF}=$ left ventricle ejection fraction; $\mathrm{LVEDD}$ = left ventricle end-diastolic diameter; sPAP = systolic pulmonary artery pressure.

On average, patients in the ADHF group had higher sPAP compared with elective patients ( $58 \pm 14 \mathrm{mmHg}$ vs. $53 \pm 15 \mathrm{mmHg}, p=0.06)$. Among patients admitted due to ADHF, 15 cases $(32.6 \%)$ had functional ischemic MR due to a recent MI. The other 22 patients $(47.2 \%)$ had functional MR due to decompensated DCM. Nine patients (19.6\%) had severe degenerative MR secondary to ruptured cord (Figure 1).

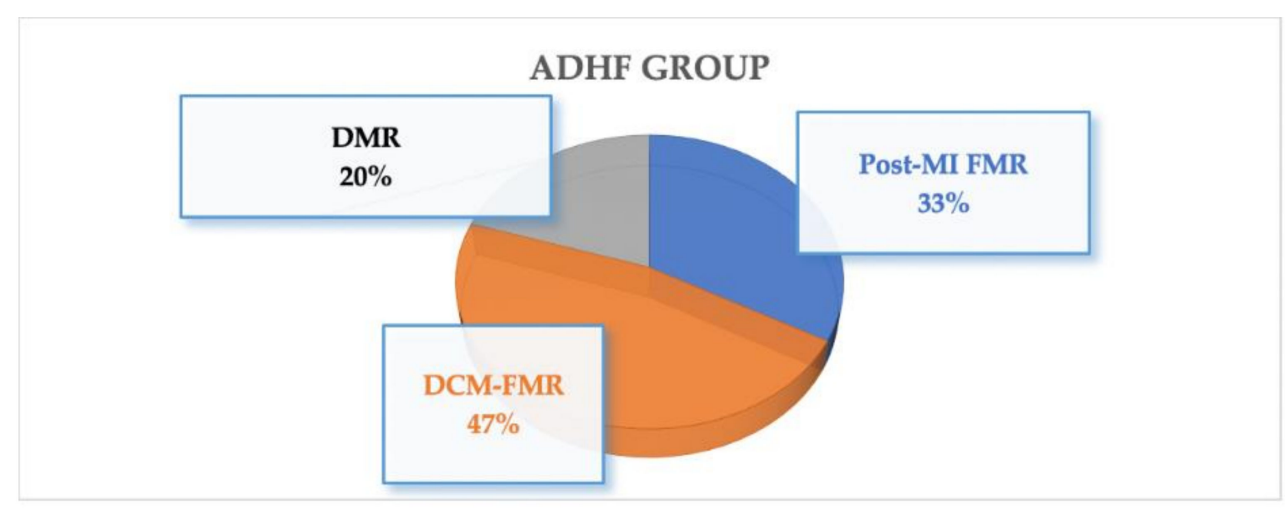

Figure 1. Etiology of $M R$ in patients admitted due to $A D H F . M R=$ mitral regurgitation; $A D H F=$ acute decompensated heart failure; $\mathrm{MI}=$ myocardial infarction; $\mathrm{DMR}=$ degenerative mitral regurgitation; $\mathrm{FMR}$ = functional mitral regurgitation; DCM = dilated cardiomyopathy. 
Reduction of MR severity was achieved in 209 out of 237 patients with device success of $89.9 \%$. Patients presented with ADHF were implanted with more clips compared with the elective group $(16 \%, 64 \%$, and $20 \%$ vs. $36 \%, 55 \%$, and $9 \%$ for one, two, and three clips, respectively, $p=0.01)$. Peri-procedural complications were observed in 14 patients $(4.6 \%)$. There were no differences in procedural success and periprocedural complications between the two groups (Table 3).

Table 3. Periprocedural characteristics of patient population underwent PMVr.

\begin{tabular}{|c|c|c|c|c|}
\hline Characteristic & $\begin{array}{l}\text { Total Population, } \\
\qquad n=237\end{array}$ & $\begin{array}{l}\text { ADHF Patients, } \\
\qquad \begin{array}{c}n=46\end{array}\end{array}$ & $\begin{array}{l}\text { Elective Patients, } \\
\qquad n=191\end{array}$ & $p$-Value \\
\hline Procedural success & $\begin{array}{c}213 / 237 \\
89.9 \%\end{array}$ & $\begin{array}{l}44 / 46 \\
91.3 \%\end{array}$ & $\begin{array}{c}169 / 191 \\
89.3 \%\end{array}$ & 0.43 \\
\hline One clip implantation, $\%$ & $\begin{array}{c}76 / 237 \\
32 \%\end{array}$ & $\begin{array}{l}7 / 46 \\
16 \%\end{array}$ & $\begin{array}{c}69 / 191 \\
36 \%\end{array}$ & \\
\hline Two-clip implantation, \% & $\begin{array}{c}135 / 237 \\
57 \%\end{array}$ & $\begin{array}{c}30 / 46 \\
64 \%\end{array}$ & $\begin{array}{c}105 / 191 \\
55 \%\end{array}$ & 0.01 \\
\hline Three-clip implantation, $\%$ & $\begin{array}{c}26 / 237 \\
11 \%\end{array}$ & $\begin{array}{l}9 / 46 \\
20 \%\end{array}$ & $\begin{array}{c}17 / 191 \\
9 \%\end{array}$ & \\
\hline Procedural Complications & $\begin{array}{c}14 / 237 \\
4.6 \%\end{array}$ & $\begin{array}{l}2 / 46 \\
4.3 \%\end{array}$ & $\begin{array}{c}12 / 191 \\
6.3 \%\end{array}$ & 0.47 \\
\hline
\end{tabular}

$\mathrm{PMVr}=$ percutaneous mitral valve repair; $\mathrm{ADHF}=$ acute decompensated heart failure.

Post-procedural echocardiography showed marked MR reduction in patients of both ADHF and elective admission groups. There was a significant mean left atrial V-wave reduction from $33 \pm 13 \mathrm{mmHg}$ to $18 \pm 8 \mathrm{mmHg}$ after the procedure $(p<0.01)$. V-wave reduction was significant in both groups. There were no signs of iatrogenic significant mitral stenosis with mean MV gradient of $4.1 \pm 2.0 \mathrm{mmHg}$. MV gradient was higher in the non-ADHF presentation group $(4.3 \pm 2.1 \mathrm{mmHg}$ vs. $3.4 \pm 1.4 \mathrm{mmHg}, p=0.01)$ (Table 4$)$.

Table 4. Hemodynamic and echocardiographic characteristics of patient population with severe MR before and immediately after PMVr.

\begin{tabular}{cccccccccc}
\hline & $\begin{array}{c}\text { Total } \\
\text { Population, } \\
\boldsymbol{n = 2 3 7}\end{array}$ & \multicolumn{4}{c}{$\begin{array}{c}\text { ADHF Patients, } \\
\boldsymbol{n}=\mathbf{4 6}\end{array}$} & \multicolumn{3}{c}{ Elective Patients, } \\
$n=191$
\end{tabular}

$\mathrm{PMVr}=$ percutaneous mitral valve repair; $\mathrm{ADHF}=$ acute decompensated heart failure; $\mathrm{MR}=$ mitral regurgitation; $\mathrm{MV}=$ mitral valve; Pre = pre-procedure; Post $=$ post-procedure.

The median clinical follow-up period was 620 days (interquartile range of 262-888 days).

There was an improvement in the clinical status of patients in the ADHF group during the immediate post-PMVr period. Continuous infusion of diuretics was discontinued in 41 out of 46 ADHF patients (89.1\%). Among critically ill patients, 14 out of 17 patients $(82.4 \%)$ were weaned from IV medications and 13 out of 15 patients $(86.7 \%)$ were successfully weaned from IABP mechanical support. There was a significantly higher rate of in-hospital death in the ADHF group as compared with elective patients $(10.9 \%$ vs. $2.6 \%, p=0.026)$ (Table 5). 
Table 5. Mortality and 1-year rehospitalizations rate of patient population that underwent PMVr.

\begin{tabular}{|c|c|c|c|c|}
\hline Mortality Rate & $\begin{array}{l}\text { Total Population, } \\
\qquad n=237\end{array}$ & $\begin{array}{c}\text { ADHF Patients, } \\
n=46\end{array}$ & $\begin{array}{l}\text { Elective Patients, } \\
\qquad n=191\end{array}$ & $p$-Value \\
\hline In-hospital mortality, $n(\%)$ & $\begin{array}{c}10 / 237 \\
4.2 \%\end{array}$ & $\begin{array}{c}5 / 46 \\
10.9 \%\end{array}$ & $\begin{array}{c}5 / 191 \\
2.6 \%\end{array}$ & 0.026 \\
\hline 30-day mortality, $n(\%)$ & $\begin{array}{c}11 / 237 \\
4.6 \%\end{array}$ & $\begin{array}{c}5 / 46 \\
10.9 \%\end{array}$ & $\begin{array}{l}6 / 191 \\
3.1 \%\end{array}$ & 0.042 \\
\hline 1-year mortality, $n(\%)$ & $\begin{array}{l}44 / 237 \\
18.6 \%\end{array}$ & $\begin{array}{l}10 / 46 \\
21.7 \%\end{array}$ & $\begin{array}{l}34 / 191 \\
17.9 \%\end{array}$ & 0.491 \\
\hline $\begin{array}{c}\text { 1-year HF-rehospitalizations, } \\
n(\%)\end{array}$ & $\begin{array}{c}53 / 237 \\
22.3 \%\end{array}$ & $\begin{array}{c}15 / 46 \\
33 \%\end{array}$ & $\begin{array}{c}38 / 191 \\
20 \%\end{array}$ & 0.09 \\
\hline
\end{tabular}

$\mathrm{PMVr}=$ percutaneous mitral valve repair; $\mathrm{ADHF}=$ acute decompensated heart failure.

After PMVr, there was an improvement of NYHA functional class in both groups of patients. There was no statistically significant changes of LVEDD in both groups at the follow-up period. There was a significant reduction of sPAP in both group of patients during follow-up: $54 \pm 15 \mathrm{mmHg}$ to $50 \pm 15$ in the non-ADHF group $(p=0.02)$ and $58 \pm 13 \mathrm{mmHg}$ to $52 \pm 12$ in the ADHF group $(p=0.02$ ) (Table 6).

Table 6. Clinical and echocardiographic outcomes of patient population with severe MR underwent PMVr.

\begin{tabular}{|c|c|c|c|c|c|c|c|}
\hline \multirow[t]{2}{*}{ Variable } & \multirow{2}{*}{$\begin{array}{c}\text { Total } \\
\text { Population, } \\
n=237\end{array}$} & \multicolumn{3}{|c|}{$\begin{array}{c}\text { ADHF Patients, } \\
\quad n=46\end{array}$} & \multicolumn{3}{|c|}{$\begin{array}{l}\text { Elective Patients, } \\
\qquad n=191\end{array}$} \\
\hline & & Pre & Follow-Up & $p$-Value & Pre & Follow-Up & $p$-Value \\
\hline $\begin{array}{l}\text { NYHA } \\
\text { functional } \\
\text { class }(0-4)\end{array}$ & $3.31 \pm 0.49$ & $3.93 \pm 0.26$ & $2.27 \pm 0.59$ & $<0.01$ & $3.31 \pm 0.49$ & $2.21 \pm 0.54$ & $<0.01$ \\
\hline LVEDD, mm & $57.2 \pm 10.0$ & $59.5 \pm 11.1$ & $55.6 \pm 7.9$ & 0.22 & $57.2 \pm 10.0$ & $57.4 \pm 10.9$ & 0.86 \\
\hline sPAP, mmHg & $55.5 \pm 14.7$ & $58.2 \pm 13.5$ & $51.9 \pm 12.1$ & 0.02 & $54.1 \pm 15.0$ & $50.1 \pm 15.1$ & 0.02 \\
\hline
\end{tabular}

$\mathrm{PMVr}=$ percutaneous mitral valve repair; $\mathrm{ADHF}=$ acute decompensated heart failure; $\mathrm{MR}=$ mitral regurgitation; $\mathrm{MV}=\mathrm{mitral}$ valve; Pre = pre-procedure; NYHA = New York Heart Association; LVEDD = left ventricle end-diastolic diameter; $\mathrm{sPAP}=$ systolic pulmonary artery pressure.

Thirty-day mortality rate was higher for patients presenting with ADHF compared to patients with elective admission $(10.9 \%$ vs. $3.1 \%, p=0.042)$. One-year mortality rate was similar in both groups of patients $(21.7 \%$ vs. $17.9 \%, p=0.4)$ (Table 5, Figure 2$)$.

In addition, there was a tendency toward a higher 1-year HF rehospitalizations rate in the ADHF group as compared with elective patients ( $33 \%$ vs. $20 \%$ ) (Table 5, Figure 3).

Among the ADHF group, patients with acute presentation (degenerative rupture cord or acute MI) had better prognosis as compared to the acute-on-chronic DCM group (Figure 4).

Multivariable Cox regression analysis demonstrated that EuroSCORE II and sPAP at baseline were independent predictors of increased mortality at 30 days (Table 7). The only significant parameter predicting 1-year mortality was EuroSCORE II (HR 1.03, CI 1-1.05, $p=0.05$ ) (Table S1). Importantly, EuroSCORE II was higher among patients with acute presentation and might impact their outcomes. 
a.

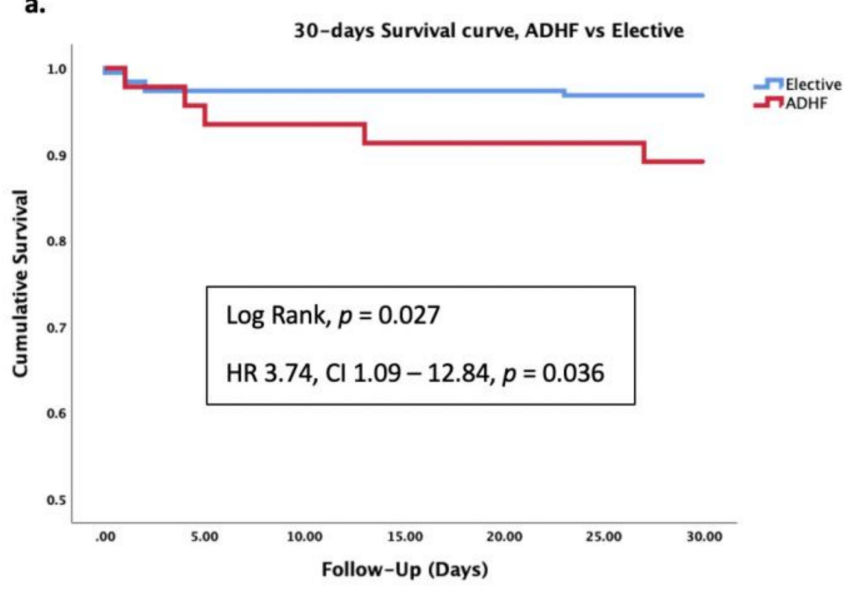

\begin{tabular}{|c|c|c|c|c|}
\hline $\begin{array}{c}\text { Number at } \\
\text { Risk }\end{array}$ & $\mathbf{0}$ & 10-days & 20-days & 30-day \\
\hline Elective & 191 & 185 & 184 & 183 \\
\hline ADHF & 46 & 43 & 42 & 40 \\
\hline
\end{tabular}

b.
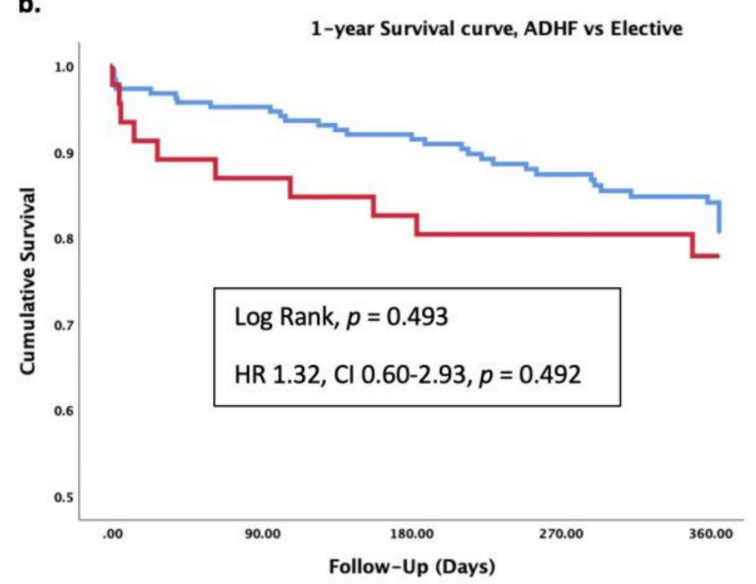

\begin{tabular}{|c|c|c|c|c|c|}
\hline $\begin{array}{c}\text { Number at } \\
\text { Risk }\end{array}$ & $\mathbf{0}$ & $\begin{array}{c}\text { 3- } \\
\text { Month }\end{array}$ & $\begin{array}{c}\text { 6- } \\
\text { Month }\end{array}$ & $\begin{array}{c}\text { 9- } \\
\text { Month }\end{array}$ & 1-Year \\
\hline Elective & 191 & 179 & 168 & 140 & 120 \\
\hline ADHF & 46 & 39 & 37 & 35 & 29 \\
\hline
\end{tabular}

Figure 2. Kaplan-Meier survival analysis of 237 patients who underwent PMVr for severe MR between 2015 and 2020 by the ADHF group and elective patients. $p$-value for log rank test. (a). 30-day analysis. (b). One-year analysis. PMVr $=$ percutaneous mitral valve repair; $\mathrm{ADHF}=$ acute decompensated heart failure; $\mathrm{MR}=$ mitral regurgitation.

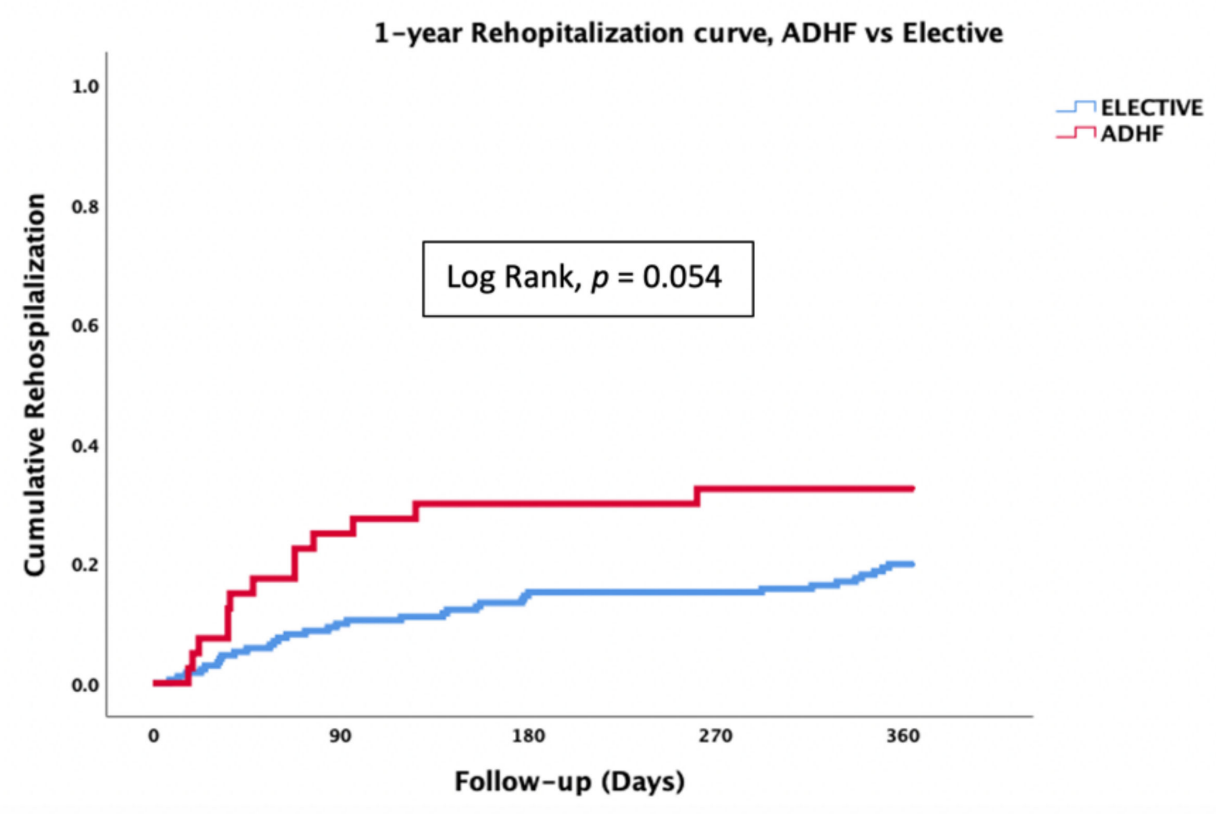

Figure 3. Kaplan-Meier analysis for 1-year HF-related rehospitalizations of 237 patients who underwent PMVr for severe MR between 2015 and 2020 by the ADHF group and elective patients $(p=0.054) . \mathrm{ADHF}=$ acute decompensated heart failure. 


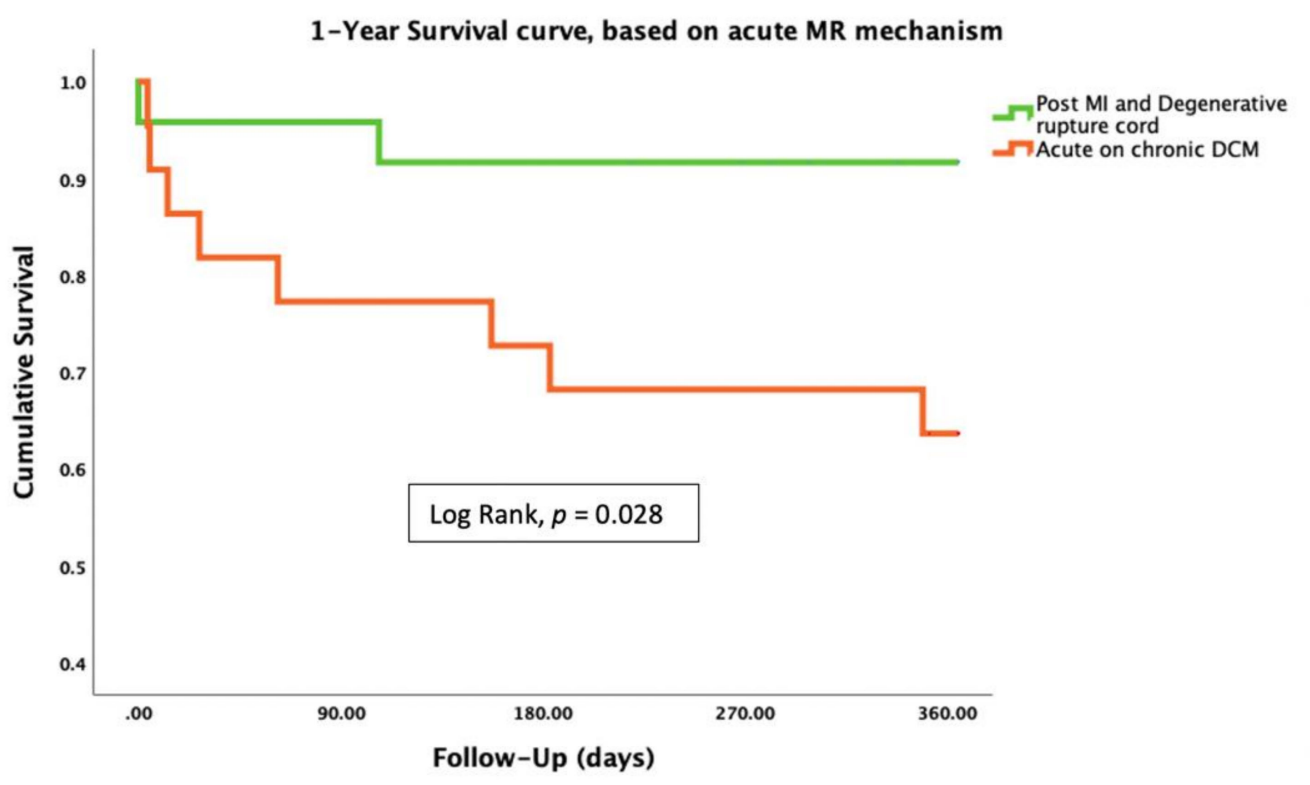

Figure 4. Kaplan-Meier survival analysis of 46 ADHF patients who underwent PMVr for severe MR between 2015 and 2020 by MR etiology. $p$-value for log rank test. PMVr = percutaneous mitral valve repair; $\mathrm{ADHF}=$ acute decompensated heart failure; $\mathrm{MR}=$ mitral regurgitation; $\mathrm{MI}=$ myocardial infarction; $\mathrm{DMR}$ = degenerative mitral regurgitation; $\mathrm{DCM}=$ dilated cardiomyopathy.

Table 7. Logistic regression analysis for prediction of cardiovascular mortality during 30-day follow-up after PMVr.

\begin{tabular}{|c|c|c|c|c|c|c|}
\hline \multirow{2}{*}{ Variable } & \multicolumn{3}{|c|}{ Univariable } & \multicolumn{3}{|c|}{ Multivariable } \\
\hline & $\mathbf{R} \mathbf{R}$ & CI & $p$-Value & $\mathbf{R R}$ & CI & $p$-Value \\
\hline Acute Clip & 3.74 & $1.01-12.84$ & 0.04 & 4.85 & $0.4-55.1$ & 0.2 \\
\hline Age & 1.01 & $0.94-1.07$ & 0.95 & & & \\
\hline Gender & 1.21 & $0.36-4.07$ & 0.76 & & & \\
\hline BMI & 1.06 & $0.97-1.16$ & 0.22 & & & \\
\hline Diabetes Mellitus & 1.05 & $0.29-3.82$ & 0.94 & & & \\
\hline Hyperlipidemia & 1.20 & $0.25-5.73$ & 0.82 & & & \\
\hline Smoker & 1.14 & $0.52-2.50$ & 0.75 & & & \\
\hline COPD & 1.17 & $0.14-9.71$ & 0.89 & & & \\
\hline CAD & 2.10 & $0.55-8.03$ & 0.28 & & & \\
\hline S/P MI & 1.67 & $0.43-6.48$ & 0.43 & & & \\
\hline CABG & 1.34 & $0.32-5.51$ & 0.69 & & & \\
\hline EuroSCORE II & 1.05 & $1.01-1.09$ & 0.02 & 1.08 & $1.02-1.14$ & $<0.01$ \\
\hline Base NYHA FC 4 & 2.95 & $0.72-12.1$ & 0.1 & 1.6 & $0.11-21.9$ & 0.72 \\
\hline Mechanism (DMR) & 0.81 & $0.20-3.32$ & 0.77 & & & \\
\hline LVEF (Grade) & 0.81 & $0.51-1.28$ & 0.37 & & & \\
\hline sPAP & 1.05 & $1.0-1.1$ & 0.05 & 1.06 & $1.01-1.12$ & 0.02 \\
\hline
\end{tabular}

$\mathrm{PMVr}=$ percutaneous mitral valve repair; $\mathrm{MI}=$ myocardial infarction; $\mathrm{BMI}=$ body mass index; $\mathrm{COPD}=$ chronic obstructive pulmonary disease; $\mathrm{CAD}=$ coronary artery disease; NYHA FC = New York Heart Association functional class; CABG = coronary artery bypass graft; $\mathrm{DMR}=$ degenerative mitral regurgitation; $\mathrm{LVEF}=$ left ventricle ejection fraction; $\mathrm{sPAP}=$ systolic pulmonary artery pressure. 


\section{Discussion}

Advances in both surgical and catheter-based therapies resulted in the extension of interventional treatments to the older, sicker population of patients with MR [4].

Recent data suggests that PMVr may be considered for elective high-risk patients with severe symptomatic functional and degenerative $\mathrm{MR}$ and $\mathrm{HF}$ as an alternative to surgical valve repair [4,12-14].

The role of PMVr is less known in the management of high-risk patients with ADHF and severe MR. In our study, we evaluated the impact of PMVr on outcomes of patients with severe MR admitted due to ADHF that required urgent PMVr and compared them with patients who underwent elective PMVr. Although short-term mortality was higher among ADHF population, 1-year mortality was similar between the groups.

In addition, we showed that PMVr during the index hospitalization is safe and effective in high-risk ADHF patients with good procedural success. It is interesting to note that in the ADHF group, despite receiving more clips, the MV gradients were lower. We might suggest that these patients have dilated ventricles with more dilated annulus. Therefore, the MV gradients were lower even with more clips implanted as compared with elective patients.

Previously, we showed that the PMVr could be a "bail-out" option in treatment of critically ill patients with refractory HF and severe functional MR [15]. In the current study, PMVr allowed to stabilize patients with ADHF and functional MR after recent MI as well as patients with acute degenerative MR who presented with ruptured cord.

Several studies evaluated the impact of MR severity, etiology, and persistency on outcomes of patients with ADHF.

The recent ARIC study showed that the higher MR severity was associated with worse prognosis in patients ADHF with excess 1-year mortality. However, a limitation in this study is the lack of differentiation between the underlying etiology of MR (primary vs. secondary/functional), though the authors suggest that a substantial portion of MR must have been functional [16].

Kubo et al. showed that in patients hospitalized for ADHF, dynamic severe MR on hospital arrival was associated with poorer outcomes than non-significant MR and had similar risk to persistent severe MR. The risk of dynamic MR was consistent in the subgroups of patients with reduced $(<45 \%)$ and preserved LV ejection fraction [17].

In our study, we evaluated patients with severe MR of either functional or degenerative etiology. We showed that the ADHF group of patients presented with acute MR (degenerative ruptured cord or post-MI) had better prognosis as compared to patients with acute-on-chronic DCM.

Impact of acute ischemic functional MR on prognosis of patients with acute MI was evaluated by Estevez-Loureiro (EREMMI registry) [18] and by Haberman et al. [19,20], which showed immediate reduction in left atrial V-wave, pulmonary artery pressure, improvement in NYHA functional class, and improved hemodynamic parameters after PMVr with MitraClip implantation.

Tomás Benito-González et al. examined procedural and clinical outcomes among 85 patients in the single center population undergoing PMVr within an admission for ADHF. In line with our findings, they found MR reduction and NYHA functional class improvement with no differences in 1-year mortality between urgent or elective PMVr groups. Interestingly, contrary to our findings, 30-day mortality was also similar between the groups; however, that might be related to a small cohort, which was underpowered to detect the survival differences [21].

Recently, Richard G Jung et al. conducted multicenter analysis and showed that PMVr may improve short- and intermediate-term mortality in high-risk patients with cardiogenic shock and moderate-to-severe MR. Patients presented with cardiogenic shock and functional MR benefit from early intervention with TEER [22]. In addition, Haberman D. et al. showed that early intervention with TEER might mitigate the poor prognosis associated with conservative therapy in patients with post-MI MR. PMVr can serve as an alternative for surgery in reducing MR for high-risk patients [23]. 
The limitations of our study include its observational retrospective nature in a twocenter population. The groups were not randomized into the different treatment arms. Additional clinical (such as NT-Pro-BNP), echocardiographic data (such as EROA) was not fully available. There was non-adherence to GDMT even in a large fraction of elective patients. In our study, critically ill patients were treated with inotropic dose of dopamine to increase blood pressure as well as IABP for mechanical support, which is not a recommended therapy for cardiogenic shock. According to recent guidelines, routine IABP use in cardiogenic shock is contraindicated, except in cases of mechanical MI complications such as acute mitral regurgitation or ventricular septal defect. In our study, the IABP was placed only in post-MI patients with acute ischemic functional MR. Large, multicenter and randomized trials are needed to confirm this data and eliminate the selection bias.

\section{Conclusions}

PMVr using MitraClip ${ }^{\circledR}$ therapy could be an alternative option for treatment of patients presented with ADHF and severe MR of both degenerative and functional etiology.

Supplementary Materials: The following are available online at https://www.mdpi.com/article/ 10.3390/jcm10245849/s1, Table S1: Logistic regression analysis for prediction of cardiovascular mortality during 1-year follow-up after PMVr.

Author Contributions: Conceptualization, A.T.M., D.H., M.S.; methodology, A.T.M., D.H., M.S.; validation, A.T.M. and D.H.; formal analysis, A.T.M. and D.H.; investigation, A.T.M., D.H., M.I., I.T., Y.R., A.P., S.S., O.T.; resources, A.T.M. and D.H.; data curation, A.T.M. and D.H.; writingoriginal draft preparation, A.T.M. and D.H.; writing-review and editing, A.T.M., D.H. and M.S.; visualization, H.D., R.B. and M.S.; project administration, A.T.M., D.H. and M.S. All authors have read and agreed to the published version of the manuscript.

Funding: This research received no external funding.

Institutional Review Board Statement: The study was conducted in accordance with the Declaration of Helsinki. The protocols of this study were approved by the Ethical Committee of the Hebrew University, Israel (protocol number 0072-18).

Informed Consent Statement: Informed consent was obtained from all subjects involved in the study.

Data Availability Statement: The data that supports the findings of this study are available from the corresponding author, M.S., upon reasonable request.

Acknowledgments: Dan Haberman would like to thank Jacob George for his great support and guidance.

Conflicts of Interest: The authors declare no conflict of interest.

\section{References}

1. Teerlink, J.R.; Alburikan, K.; Metra, M.; Rodgers, J.E. Acute decompensated heart failure update. Curr. Cardiol. Rev. 2015, 11, 53-62. [CrossRef] [PubMed]

2. Velazquez, E.J;; Morrow, D.A.; DeVore, A.D.; Duffy, C.I.; Ambrosy, A.P.; McCague, K.; Rocha, R.; Braunwald, E.; for the PIONEERHF Investigators. Angiotensin-Neprilysin Inhibition in Acute Decompensated Heart Failure. N. Engl. J. Med. 2019, 380, 539-548. [CrossRef] [PubMed]

3. Fonarow, G.C.; Abraham, W.T.; Albert, N.M.; Stough, W.G.; Gheorghiade, M.; Greenberg, B.H.; O'Connor, C.M.; Pieper, K.; Sun, J.L.; Yancy, C.W.; et al. Factors identified as precipitating hospital admissions for heart failure and clinical outcomes: Findings from OPTIMIZE-HF. Arch. Intern. Med. 2008, 168, 847-854. [CrossRef] [PubMed]

4. Sabbagh, A.E.; Reddy, Y.; Nishimura, R.A. Mitral Valve Regurgitation in the Contemporary Era: Insights into Diagnosis, Management, and Future Directions. JACC Cardiovasc. Imaging 2018, 11, 628-643. [CrossRef] [PubMed]

5. Nkomo, V.T.; Gardin, J.M.; Skelton, T.N.; Gottdiener, J.S.; Scott, C.G.; Enriquez-Sarano, M. Burden of valvular heart diseases: A population-based study. Lancet 2006, 368, 1005-1011. [CrossRef]

6. Stevenson, L.W.; Brunken, R.C.; Belil, D.; Grover-McKay, M.; Schwaiger, M.; Schelbert, H.R.; Tillisch, J.H. Afterload reduction with vasodilators and diuretics decreases mitral regurgitation during upright exercise in advanced heart failure. J. Am. Coll. Cardiol. 1990, 15, 174-180. [CrossRef] 
7. Dulgheru, R.; Magne, J.; Lancellotti, P.; Pierard, L.A. Dynamic Ischaemic Mitral Regurgitation and the Role of Stress Echocardiography. J. Cardiovasc. Echogr. 2013, 23, 10-17. [PubMed]

8. Senni, M.; Adamo, M.; Metra, M.; Alfieri, O.; Vahanian, A. Treatment of functional mitral regurgitation in chronic heart failure: Can we get a 'proof of concept' from the MITRA-FR and COAPT trials? Eur. J. Heart Fail. 2019, 21, 852-861. [CrossRef] [PubMed]

9. Asgar, A.W.; Mack, M.J.; Stone, G.W. Secondary mitral regurgitation in heart failure: Pathophysiology, prognosis, and therapeutic considerations. J. Am. Coll. Cardiol. 2015, 65, 1231-1248. [CrossRef]

10. Lavall, D.; Hagendorff, A.; Schirmer, S.H.; Böhm, M.; Borger, M.A.; Laufs, U. Mitral valve interventions in heart failure. ESC Heart Fail. 2018, 5, 552-561. [CrossRef]

11. Vahanian, A.; Alfieri, O.; Andreotti, F.; Antunes, M.J.; Barón-Esquivias, G.; Baumgartner, H.; Borger, M.A.; Carrel, T.P.; De Bonis, M.; Evangelista, A.; et al. ESC/EACTS Guidelines on the management of valvular heart disease (version 2012). The Joint Task Force on the Management of Valvular Heart Disease of the European Society of Cardiology and the European Association for Cardio-Thoracic Surgery. Eur. Heart J. 2012, 33, 2451-2496.

12. Feldman, T.; Kar, S.; Rinaldi, M.; Fail, P.; Hermiller, J.; Smalling, R.; Whitlow, P.L.; Gray, W.; Low, R.; Herrmann, H.C.; et al. EVEREST investigators. Percutaneous mitral repair with the MitraClip system: Safety and midterm durability in the initial EVEREST (Endovascular Valve Edge-to-Edge REpair Study) cohort. J. Am. Coll. Cardiol. 2009, 54, 686-694. [CrossRef] [PubMed]

13. Obadia, J.F.; Messika-Zeitoun, D.; Leurent, G.; Iung, B.; Bonnet, G.; Piriou, N.; Nefèvre, T.; Piot, C.; Rouleau, F.; Carrié, D.; et al. MITRA-FR Investigators. Percutaneous repair or medical treatment for secondary mitral regurgitation. N. Engl. J. Med. 2018, 379, 2297-2306. [CrossRef]

14. Stone, G.W.; Lindenfeld, J.; Abraham, W.T.; Kar, S.; Lim, D.S.; Mishell, J.M.; Whisenant, B.; Grayburn, P.A.; Rinaldi, M.; Kapadia, S.R.; et al. COAPT Investigators. Tran-scatheter mitral-valve repair in patients with heart failure. N. Engl. J. Med. 2018, 379, 2307-2318. [CrossRef] [PubMed]

15. Turyan-Medvedovsky, A.; Tonchev, I.; Tahiroglu, I.; Lotan, C.; Gilon, D.; Planer, D.; Danenberg, H.D.; Beeri, R.; Shuvy, M. MitraClip Therapy in Critically Ill Patients with Severe Functional Mitral Regurgitation and Refractory Heart Failure. Struct. J. Heart Team 2019, 3, 296-301. [CrossRef]

16. Arora, S.; Sivaraj, K.; Hendrickson, M.; Chang, P.P.; Weickert, T.; Qamar, A.; Vaduganathan, M.; Caughey, M.C.; Pandey, A.; Cavender, M.A.; et al. Prevalence and Prognostic Significance of Mitral Regurgitation in Acute Decompensated Heart Failure: The ARIC Study. JACC Heart Fail. 2021, 9, 179-189. [CrossRef] [PubMed]

17. Kubo, S.; Kawase, Y.; Hata, R.; Maruo, T.; Tada, T.; Kadota, K. Dynamic severe mitral regurgitation on hospital arrival as prognostic predictor in patients hospitalized for acute decompensated heart failure. Int. J. Cardiol. 2018, 15, 177-182. [CrossRef]

18. Estevez-Loureiro, R.; Adamo, M.; Arzamendi, D.; Denti, P.; Freixa, X.; Nombela-Franco, L.; Pascual, I.; Melica, B.; Attias, D.; Serrador, A.; et al. Transcatheter mitral valve repair in patients with acute myocardial infarction: Insights from the European Registry of MitraClip in Acute Mitral Regurgitation following an acute myocardial infarction (EREMMI). EuroIntervention 2020, 15, 1248-1250. [CrossRef] [PubMed]

19. Haberman, D.; Taramasso, M.; Czarnecki, A.; Kerner, A.; Chrissoheris, M.; Spargias, K.; Poles, L.; Agmon, Y.; Scianna, S.; Beeri, R.; et al. Salvage MitraClip in severe secondary mitral regurgitation complicating acute myocardial infarction: Data from a multicentre international study. Eur. J. Heart Fail. 2019, 21, 1161-1164. [CrossRef] [PubMed]

20. Haberman, D.; Estévez-Loureiro, R.; Benito-Gonzalez, T.; Denti, P.; Arzamendi, D.; Adamo, M.; Freixa, X.; Nombela-Franco, L.; Villablanca, P.; Krivoshei, L.; et al. Safety and Feasibility of MitraClip Implantation in Patients with Acute Mitral Regurgitation after Recent Myocardial Infarction and Severe Left Ventricle Dysfunction. J. Clin. Med. 2021, 10, 1819. [CrossRef]

21. Benito-González, T.; Estévez-Loureiro, R.; Del Castillo, S.; Minguito-Carazo, C.; Garrote-Coloma, C.; Alonso-Rodríguez, D.; Tundidor-Sanz, E.; Rodríguez-Santamarta, M.; Pérez de Prado, A.; Ramón, C.C.; et al. Clinical outcomes following urgent vs. elective percutaneous mitral valve repair. Cardiovasc. Revasc. Med. 2020, 9, S1553-S8389. [CrossRef] [PubMed]

22. Jung, R.G.; Simard, T.; Kovach, C.; Flint, K.; Don, C.; Santo, P.; Adamo, M.; Branca, L.; Valentini, F.; Benito-González, T.; et al. Transcatheter Mitral Valve Repair in Cardiogenic Shock and Mitral Regurgitation: A Patient-Level, Multicenter Analysis. JACC Cardiovasc. Interv. 2021, 14, 1-11. [CrossRef] [PubMed]

23. Haberman, D.; Estévez-Loureiro, R.; Benito-Gonzalez, T.; Denti, P.; Arzamendi, D.; Adamo, M.; Freixa, X.; Nombela-Franco, L.; Villablanca, P.; Krivoshei, L.; et al. Conservative, surgical, and percutaneous treatment for mitral regurgitation shortly after acute myocardial infarction. Eur. Heart J. 2021, 496, online ahead of print. [CrossRef] [PubMed] 